\title{
Raman microspectroscopy of nucleus and cytoplasm for human colon cancer diagnosis
}

\author{
Wenjing Liu ${ }^{\mathrm{a}, \mathrm{b}}$, Hongbo Wang ${ }^{\mathrm{c}}$, Jingjing Du ${ }^{\mathrm{a}, \mathrm{b}}$, Chuanyong Jing ${ }^{\mathrm{a}, \mathrm{b}, *}$ \\ a State Key Laboratory of Environmental Chemistry and Ecotoxicology, Research Center for Eco-Environmental Sciences, Chinese Academy of Sciences, \\ Beijing 100085, China \\ b University of Chinese Academy of Sciences, Beijing 100049, China \\ ${ }^{\mathrm{c}}$ School of Municipal and Environmental Engineering, Shandong Jianzhu University, Jinan 250101, China
}

\section{A R T I C L E I N F O}

\section{Keywords:}

Subcellular

Raman

Actual human tissue

CLS model

PLS-DA model

\begin{abstract}
A B S T R A C T
Subcellular Raman analysis is a promising clinic tool for cancer diagnosis, but constrained by the difficulty of deciphering subcellular spectra in actual human tissues. We report a label-free subcellular Raman analysis for use in cancer diagnosis that integrates subcellular signature spectra by subtracting cytoplasm from nucleus spectra (Nuc.-Cyt.) with a partial least squares-discriminant analysis (PLS-DA) model. Raman mapping with the classical least-squares (CLS) model allowed direct visualization of the distribution of the cytoplasm and nucleus. The PLS-DA model was employed to evaluate the diagnostic performance of five types of spectral datasets, including non-selective, nucleus, cytoplasm, ratio of nucleus to cytoplasm (Nuc./Cyt.), and nucleus minus cytoplasm (Nuc.-Cyt.), resulting in diagnostic sensitivity of $88.3 \%, 84.0 \%, 98.4 \%, 84.5 \%$, and $98.9 \%$, respectively. Discriminating between normal and cancerous cells of actual human tissues through subcellular Raman markers is feasible, especially when using the nucleus-cytoplasm difference spectra. The subcellular Raman approach had good stability, and had excellent diagnostic performance for rectal as well as colon tissues. The insights gained from this study shed new light on the general applicability of subcellular Raman analysis in clinical trials.
\end{abstract}

\section{Introduction}

Confocal Raman microspectroscopy (CRM) has been demonstrated to be a promising clinical tool for cancer diagnosis and imaging (Kendall et al., 2009; Abramczyk and Brozek-Pluska, 2013; Liu et al., 2012; Kong et al., 2013). With the help of fast-developing imaging techniques, CRM mapping can differentiate various subcellular compartments of individual cells (Tsai et al., 2014). CRM enables an in situ view of biochemical changes during cellular metabolism, transformation, and senescence (Duraipandian et al., 2013; Lin et al., 2009).

The importance of subcellular Raman analysis in cancer diagnosis by CRM has only recently been realized (Farhane et al., 2015a, 2015b; Piredda et al., 2015; Carvalho et al., 2015; Hewitt et al., 2015). For example, Raman spectra of the nucleoli, nuclei, and cytoplasm from oral cell lines were collected and independently processed by principal component analysis (PCA) to discriminate cancer from normal cell lines (Carvalho et al., 2015). In addition, Piredda et al. proposed a subcellular approach in cancer diagnosis by using the peak ratio of nucleus (nucleic acid) and cytoplasm (tryptophan) in skin model cells
(Piredda et al., 2015). The peak ratio was employed to define a decision boundary criterion for cancer diagnosis. However, the arbitrary classification obtained using these simple ratios might be biased because the criterion value was strongly dependent on the model cells, thus highly restraining its application in other model cells and human cytological samples.

The human samples for conventional biopsy and histopathological analysis in clinical diagnosis are much more complex in differentiation grade and composition than model cells. Meanwhile, large numbers of human samples are needed to build a generalized model for cancer diagnosis, considering the intra- and inter-patient variabilities. To the best of our knowledge, however, no subcellular Raman analysis for cancer diagnosis has been performed using human tissue samples. Therefore, there is an urgent need to analyze actual tissues at the subcellular level to bridge laboratory and clinical trials.

Our motivation is to propose a generalized subcellular Raman approach in cancer diagnosis for actual human tissues. Our hypothesis is that this goal will be fulfilled by combining subcellular signature spectra derived by subtracting the cytoplasm and nucleus spectra

\footnotetext{
* Corresponding author at: State Key Laboratory of Environmental Chemistry and Ecotoxicology, Research Center for Eco-Environmental Sciences, Chinese Academy of Sciences, Beijing 100085, China.

E-mail address: cyjing@rcees.ac.cn (C. Jing).
} 
(Nuc.-Cyt.) with a partial least squares-discriminant analysis (PLS-DA) multivariate algorithm. Integrating the Raman spectra of the nucleus and cytoplasm can deliver comprehensive information for cells. Moreover, the PLS-DA model has been successfully applied in Raman spectral analysis, providing statistically reliable diagnosis results (Duraipandian et al., 2012). As a proof-of-concept study, we analyzed Raman spectra and images of human colon tissues to establish a standardized methodology to differentiate the normal and cancer cells. The insights gained from this study shed new light on the general applicability of subcellular Raman analysis in clinical trials.

\section{Materials and methods}

\subsection{Sample preparation}

Formalin-fixed, paraffin-embedded colon tissues from 188 persons were retrieved from the Jinan No. 4 Hospital in accordance with the regulations of its ethics committee. The Jinan No. 4 Hospital has approved this study. The paraffin-embedded tissues were sectioned into $10 \mu \mathrm{m}$ thick sections. Sections were placed on a glass microscope slides in adjacent pairs, with one section of each pair stained with hematoxylin and eosin (H \& E) for histopathological diagnosis of the suspected area, and the adjacent section left unstained for Raman spectroscopy analysis (Salman et al., 2015; Argov et al., 2002). The histological analysis was conducted by professional medical doctors who are board certified as pathologists.

\subsection{Confocal Raman spectroscopy and imaging}

Raman spectra were acquired with a $10 \mathrm{~s}$ integration time in the spectral range of $400-4000 \mathrm{~cm}^{-1}$ using a Raman system (Horiba JY HR evolution, France). A $532 \mathrm{~nm}$ laser was focused through a $100 \times$ objective $(\mathrm{NA}=0.9, \mathrm{WD}=0.21 \mathrm{~mm})$ to excite the samples. The PLS-DA model was applied for Raman spectra analysis. Raman images $(120 \times$ $120 \mu \mathrm{m}, 25 \times 25$ points) of colon tissue were constructed in the spectral region $800-1800 \mathrm{~cm}^{-1}$ by the Raman system. Labspec6 software (Horiba JY) was utilized for preprocessing each individual Raman spectrum. The classical least-squares (CLS) model was utilized to analyze Raman imaging of normal and cancerous tissues. In addition, the adjusted Rand index (ARI) was calculated to assess the robustness of Non-selective, Nucleus, Cytoplasm, Nuc./Cyt., and Nuc.-Cyt methods. The detailed information regarding spectra collection, model analysis, and approach assessment can be seen in the Supplementary material.

\section{Results and discussion}

\subsection{Subcellular Raman spectra of colon tissues}

Averaged Raman spectra of normal and cancerous tissues are shown in Fig. 1A, and the peak assignments are listed in Table S1. The spectra provide indistinguishable information for normal and cancerous cells, suggesting that the averaged spectra cannot be used for classification purposes. This lack of classification could be solved considering the stark contrast between nucleus and cytoplasm on the $\mathrm{H} \& \mathrm{E}$ staining images (Fig. 1B), where cell nuclei (navy blue) were scattered within the cytoplasm (pink). Raman spectra were then collected from unstained samples for the nucleus and cytoplasm regions accordingly. Fig. $1 \mathrm{C}$ and $\mathrm{D}$ show representative spectra of nucleus and cytoplasm in normal and cancerous tissues. The Raman bands, due to the intrinsic vibrational modes of biomolecules, were mainly observed in the fingerprint $\left(800-1800 \mathrm{~cm}^{-1}\right)$ and high-wavenumber regions $\left(2500-3200 \mathrm{~cm}^{-1}\right.$ ) (Duraipandian et al., 2012; Bergholt et al., 2011; Lin et al., 2012). Unfortunately, the differences between these components in normal and cancerous tissues are too subtle to achieve cancer diagnosis. Interestingly, the subtle differences became substantial when comparing the differential Raman spectra of nucleus and cytoplasm (Nuc.-Cyt.) between normal and cancerous tissues, especially in the fingerprint region (Fig. 1E).

\subsection{Validation of subcellular regions using Raman mapping}

The recognition of nucleus and cytoplasm from unstained images, which is the foundation of subcellular analysis, might be biased and arbitrary if not rigorously verified. To justify the definition of nucleus and cytoplasm in human colon cells, photomicrographs of $\mathrm{H} \& \mathrm{E}$ stained and unstained tissue sections for normal and cancerous cells were collected as shown in Fig. 2A, B, F, G. Direct comparison for Raman maps in the 1049-1076 $\mathrm{cm}^{-1}$ region for normal cells (Fig. 2C) and cancerous cells (Fig. $2 \mathrm{H}$ ) was not able to clearly differentiate the nucleus and cytoplasm. Therefore, multivariate analysis was performed using several widely-used models on the Raman mapping data in the fingerprint region.

Classical least-squares (CLS), multivariate curve resolution (MCR), hierarchical clustering analysis (HCA), and divisive clustering analysis (DCA) were employed. As shown in Fig. S3, the CLS model resulted in the highest spatial resolution as compared with the other three models. In agreement with a previous report (Keating et al., 2012), CLS is clearly a powerful technique to analyze the subcellular structure, and therefore it was used to analyze Raman maps. Upon data processing using the CLS model as detailed in the SI, the Raman mapping images for normal and cancerous cells (Fig. 2D, I) were obtained. The corresponding spectra of nucleus and cytoplasm are shown in Fig. 2E, J. A comparison of the spectra from nucleus and cytoplasm reveals that the most notable difference between the spectra lies in the vibrational bands at 1060 and $1292 \mathrm{~cm}^{-1}$ associated with $\mathrm{C}-\mathrm{C}$ stretching (lipids), and $\mathrm{C}-\mathrm{H}_{2}$ twisting (phospholipids), which is more intense in the nucleus spectra. The CLS analysis showed that the distribution of the nucleus and cytoplasm in the normal and cancerous cells corresponded with the H \& E stained images. Thus, Raman mapping results with CLS fitting can be regarded as supporting evidence to resolve subcellular structure.

\subsection{Multivariate spectral analysis and diagnostics}

The partial least squares-discriminant analysis (PLS-DA) multivariate algorithm, with leave-one-patient-out cross validation, was employed to analyze the Raman spectra using five sets of spectral data, namely Non-selective, Nucleus, Cytoplasm, Nuc./Cyt., and Nuc.-Cyt..

In the PLS-DA model, the selected latent variable (LV) number was $5,7,4,2$, and 2, corresponding to the five Raman spectral datasets (Table S2), and their selection rationale is detailed in the SI.

The box charts of significant LV scores obtained from all spectral datasets were used to visualize the different degrees of diagnostic utility of significant LVs for dysplasia classification (Figs. S4-S6).

To examine the applicability of the five datasets, a receiver operating characteristic (ROC) curve was constructed as shown in Fig. S7. The area under curve (AUC) for the five datasets was in the range $0.92-0.99$, higher than the required value of 0.90 , suggesting that all five methods could be used for clinical trials (Hanley and McNeil, 1982). The posterior probability scatter plots, as shown in Fig. S8, clearly witnessed the diagnostic efficiency using the five datasets for selecting the best methods. As a result, the Nuc.-Cyt. dataset yielded the diagnostic sensitivity of $98.9 \%$ and specificity of $97.0 \%$, which is the best among the five datasets (Table 1). This result was as sensitive as the subcellular method using model cells, even though model cells usually lead to high sensitivity because of their simple and uniform composition, suggesting that our subcellular method is promising for use in clinical trials.

As the PLS-DA diagnostic results were slightly influenced by the variabilities between patients (Table S3), the robustness of the five diagnostic methods was assessed by ARI (Fig. S9). A high ARI value 


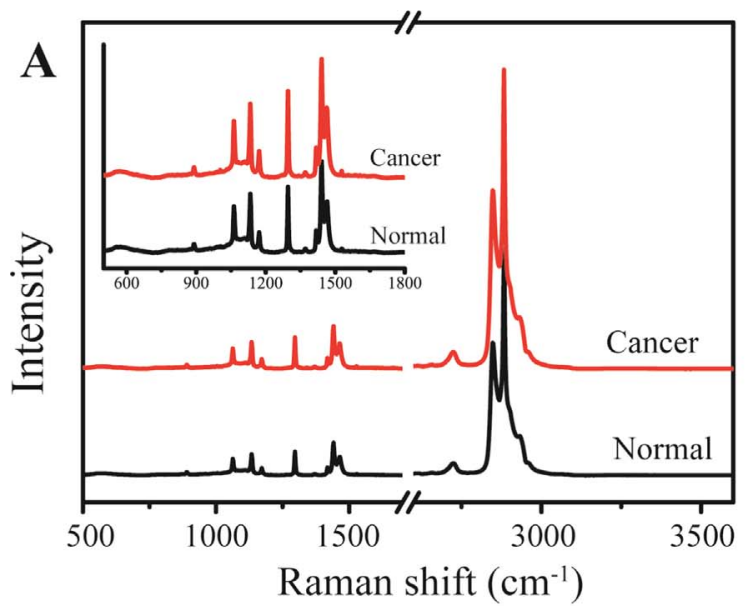

B
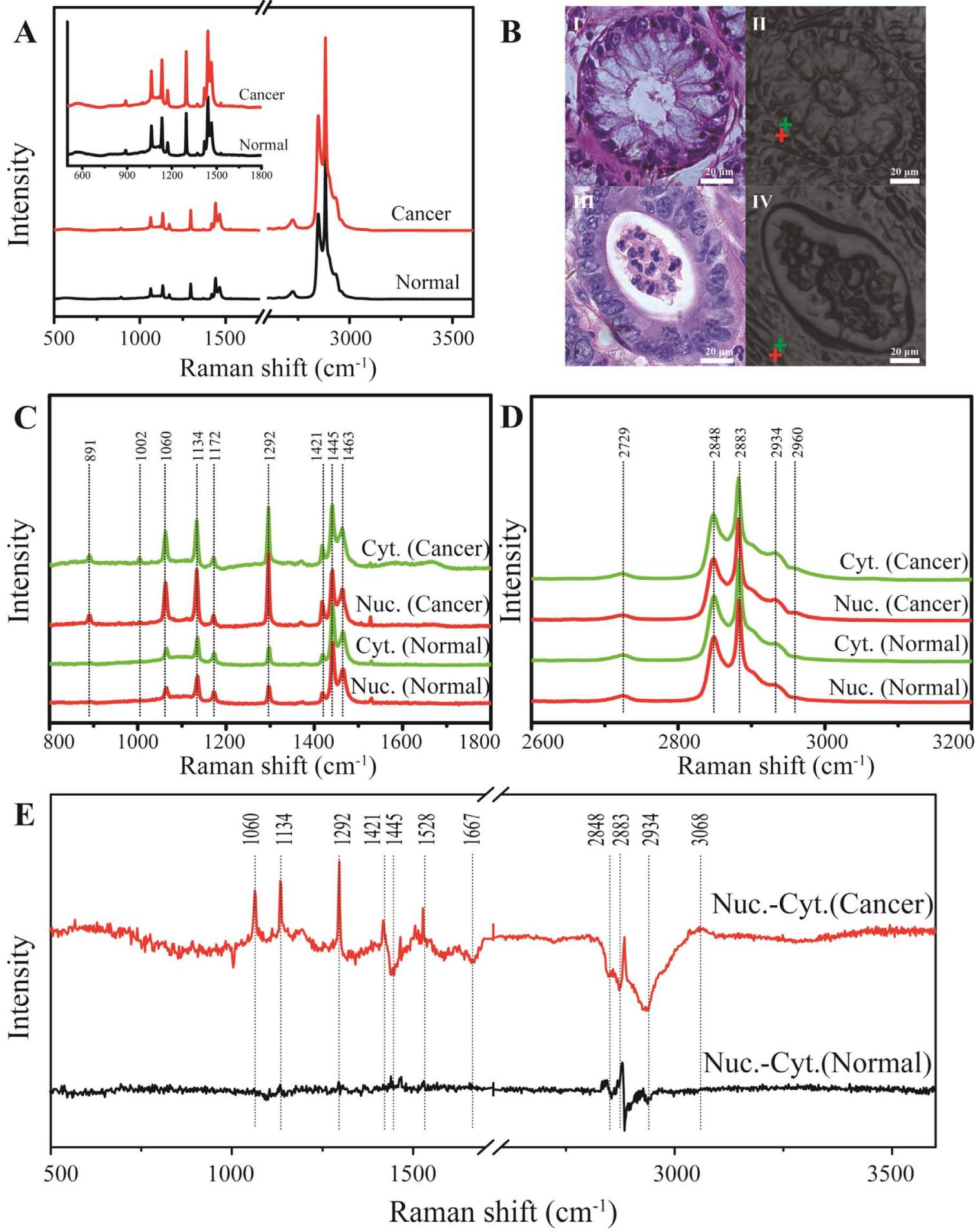

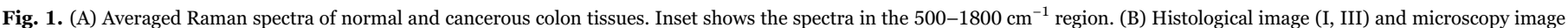

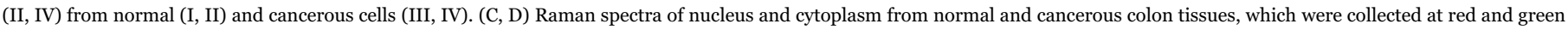

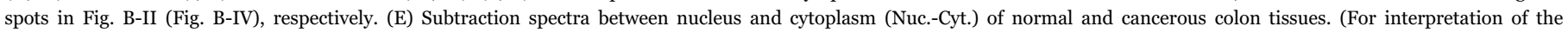
references to color in this figure legend, the reader is referred to the web version of this article.)

implies that the method is robust with respect to data perturbation (Hubert and Arabie, 1985; Yeung et al., 2003). All pairwise comparisons of ARI values were statistically significant, as evidenced by $\mathrm{p}<$ 0.05 using one-way ANOVA and least significant difference (LSD) analysis. The average ARI value of Nuc.-Cyt. (0.921) was significantly higher than Non-selective (0.737), Nucleus (0.765), Cytoplasm (0.816), and Nuc./Cyt. (0.885), suggesting that Nuc.-Cyt. is the most stable method.
The diagnostic approach was extended to rectal tissue to test its applicability for human cancer diagnosis. A total of 260 Raman spectra were collected from 65 tissues corresponding to 65 patients. Five spectral datasets, including Non-selective, Nucleus, Cytoplasm, Nuc./ Cyt., and Nuc.-Cyt. were analyzed by the PLS-DA model with the selected LV numbers of 3, 4, 3, 3, and 7, respectively (Table S4). As shown in Table S5, Nuc.-Cyt. yielded the highest diagnostic sensitivity (93.1\%) and specificity (91.1\%). This result demonstrates the potential 

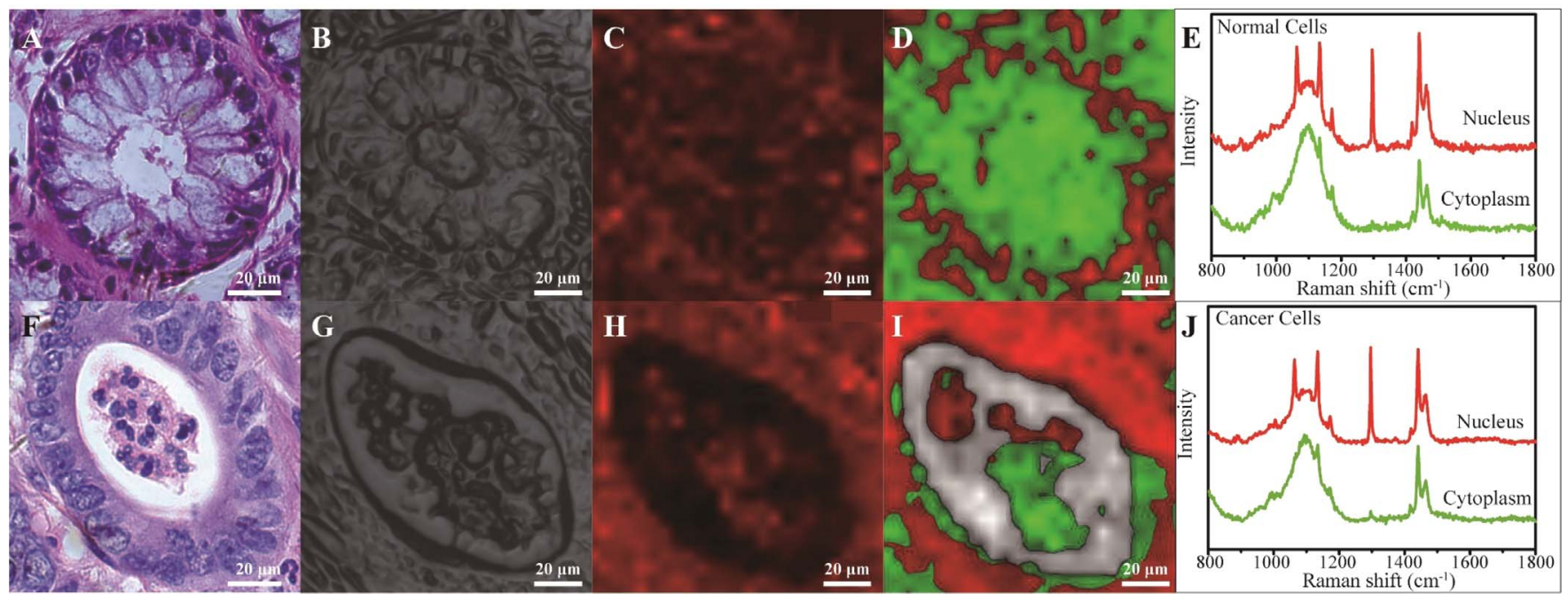

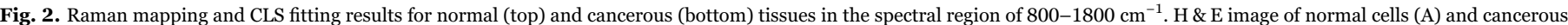

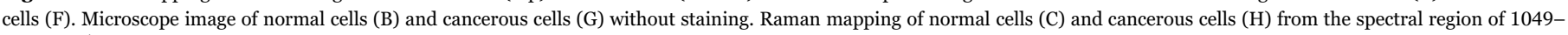

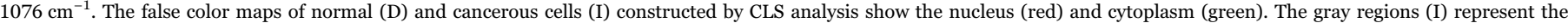

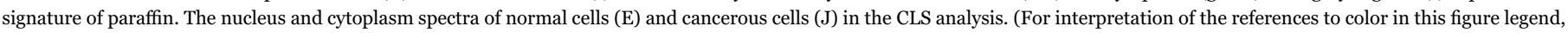
the reader is referred to the web version of this article.)

Table 1

Comparison of diagnostic performance of different methods for classifying colon tissues using PLS-DA together with leave-one-patient-out cross-validation.

\begin{tabular}{llll}
\hline Methods & LVs & Sensitivity (\%) & Specificity (\%) \\
\hline Non-selective & 5 & $88.3(166 / 188)$ & $89.5(179 / 200)$ \\
Nucleus & 7 & $84.0(158 / 188)$ & $87.0(174 / 200)$ \\
Cytoplasm & 4 & $98.4(185 / 188)$ & $97.5(195 / 200)$ \\
Nuc./Cyt. & 2 & $84.5(159 / 188)$ & $90.0(180 / 200)$ \\
Nuc.-Cyt. & 2 & $98.9(186 / 188)$ & $97.0(194 / 200)$ \\
\hline
\end{tabular}

applicability of our PLS-DA Raman microspectroscopy approach for human cancer diagnosis.

The variance of subcellular regions (nucleus and cytoplasm) between normal and cancer cells is larger than that of whole cellular region (non-selective). Cancer cells are continually undergoing transformation to enable their survival, and some significant changes can be easily detected by Raman spectroscopy (Farhane et al., 2015b). However, some crucial changes, such as mutations of the many genes encoding proteins that govern processes of cell proliferation, differentiation, and development, resulting in the change of cell components (Sherr, 2004), are an inherent challenge to non-selective Raman analysis due to its low sensitivity. This difficulty is successfully solved using subcellular Raman analysis (Farhane et al., 2015a). Therefore, the approaches using nucleus, cytoplasm, Nuc./Cyt., and Nuc.-Cyt. are superior to the non-selective analysis.

In agreement with previous reports (Duraipandian et al., 2013; Terentis et al., 2013), higher sensitivity and specificity were obtained from cytoplasm than nucleus (Table 1). Cell proliferation requires nutrients, energy, and biosynthetic activity to duplicate all macromolecular components. The biosynthesis of increased amounts of macromolecules mainly occurs in the cytoplasmic region (DeBerardinis et al., 2008). In addition, increased mRNA translation occurred during the differentiation in the cytoplasm (Ghita et al., 2012). Therefore, the chemical change in cytoplasm was more significant than that in the nucleus.

Although the cytoplasm method could yield acceptable diagnostic sensitivity and specificity, Nuc.-Cyt. includes the whole cell information, resulting in a more accurate diagnosis. The spectral profiles with the information from the nucleus and cytoplasm can decrease the model perturbation when facing the variabilities between patients in clinical trials. Thus, the Nuc.-Cyt. method with comprehensive cell information is much more stable than other methods. Meanwhile, Nuc.-Cyt. method is readily applicable in other tissues such as those from the rectum.

\section{Conclusion}

In summary, we proposed the subcellular subtraction spectra of the nucleus and cytoplasm (Nuc.-Cyt.) as a straightforward Raman signature for colon tumor diagnosis. PLS-DA analysis for the subtraction spectra resulted in the diagnostic sensitivity of $98.9 \%$ and specificity of 97.0\%. The results of this work suggest that our subcellular Raman analysis approach might be a powerful tool in improving cancer diagnosis during clinical examination.

\section{Acknowledgments}

We acknowledge the financial support of the National Basic Research Program of China (2014CB441102 and 2016YFA0203102), the Strategic Priority Research Program of the Chinese Academy of Sciences (XDB14020201), the National Natural Science Foundation of China (41425016, 21321004, and 21337004).

\section{Appendix A. Supplementary material}

Supplementary data associated with this article can be found in the online version at http://dx.doi.org/10.1016/j.bios.2017.05.045.

\section{References}

Abramczyk, H., Brozek-Pluska, B., 2013. Chem. Rev. 113, 5766-5781.

Argov, S., Ramesh, J., Salman, A., Sinelnikov, I., Goldstein, J., Guterman, H., Mordechai, S., 2002. J. Biomed. Opt. 7, 248-254.

Bergholt, M.S., Zheng, W., Lin, K., Ho, K.Y., Teh, M., Yeoh, K.G., So, J.B.Y., Huang, Z., 2011. Biosens. Bioelectron. 26, 4104-4110.

Carvalho, L.F.C.S., Bonnier, F., O'Callaghan, K., O'Sullivan, J., Flint, S., Byrne, H.J., Lyng, F.M., 2015. Exp. Mol. Pathol. 98, 502-509.

DeBerardinis, R.J., Lum, J.J., Hatzivassiliou, G., Thompson, C.B., 2008. Cell Metab. 7, 11-20.

Duraipandian, S., Zheng, W., Ng, J., Low, J.J.H., Ilancheran, A., Huang, Z., 2012. Anal. Chem. 84, 5913-5919.

Duraipandian, S., Zheng, W., Ng, J., Low, J.J.H., Ilancheran, A., Huang, Z., 2013. Analyst $138,4120-4128$

Farhane, Z., Bonnier, F., Casey, A., Byrne, H.J., 2015a. Analyst 140, 4212-4223. Farhane, Z., Bonnier, F., Casey, A., Maguire, A., O'Neill, L., Byrne, H.J., 2015b. Analyst 
140, 5908-5919.

Ghita, A., Pascut, F.C., Mather, M., Sottile, V., Notingher, I., 2012. Anal. Chem. 84, 3155-3162.

Hanley, J.A., McNeil, B., 1982. J. Radiol. 143, 29-36.

Hewitt, K.C., Rad, J.G., McGregor, H.C., Brouwers, E., Sapp, H., Short, M.A., Fashir, S.B., Zeng, H., Alwayn, I.P., 2015. Analyst 140, 6602-6609.

Hubert, L., Arabie, P., 1985. J. Classif. 2, 193-218.

Kendall, C., Isabelle, M., Bazant-Hegemark, F., Hutchings, J., Orr, L., Babrah, J., Baker, R., Stone, N., 2009. Analyst 134, 1029-1045.

Kong, K., Rowlands, C.J., Varma, S., Perkins, W., Leach, I.H., Koloydenko, A.A.,

Williams, H.C., Notingher, I., 2013. Proc. Natl. Acad. Sci. USA 110, 15189-15194.

Lin, J., Chen, R., Feng, S., Li, Y., Huang, Z., Xie, S., Yu, Y., Cheng, M., Zeng, H., 2009.
Biosens. Bioelectron. 25, 388-394.

Lin, K., Cheng, D.L.P., Huang, Z., 2012. Biosens. Bioelectron. 35, 213-217.

Liu, H., Zhao, J., McLean, D., Zeng, H., 2012. Cancer Rev. 10, 2491-2500.

Piredda, P., Berning, M., Boukamp, P., Volkmer, A., 2015. Anal. Chem. 87, 6778-6785. Salman, A., Sebbag, G., Argov, S., Mordechai, S., Sahu, R.K., 2015. J. Biomed. Opt. 20, 075007.

Sherr, C.J., 2004. Cell 116, 235-246.

Terentis, A.C., Fox, S.A., Friedman, S.J., Spencer, E.S., 2013. J. Raman Spectrosc. 44 1205-1216.

Tsai, T.-H., Short, M.A., McLean, D.I., Zeng, H., McElwee, K., Lui, H., 2014. Analyst 139, 2799-2805.

Yeung, K.Y., Medvedovic, M., Bumgarner, R.E., 2003. Genome Biol. 4 (R34), 1-17. 